Homology, Homotopy and Applications, vol. 15(2), 2013, pp.83-102

\title{
POWER MAPS ON $p$-REGULAR LIE GROUPS
}

\author{
STEPHEN THERIAULT
}

\author{
(communicated by Donald M. Davis)
}

\begin{abstract}
A simple, simply-connected, compact Lie group $G$ is $p$ regular if it is homotopy equivalent to a product of spheres when localized at $p$. If $A$ is the corresponding wedge of spheres, then it is well known that there is a $p$-local retraction of $G$ off $\Omega \Sigma A$. We show that that complementary factor is very well behaved, and this allows us to deduce properties of $G$ from those of $\Omega \Sigma A$. We apply this to show that, localized at $p$, the $p^{\text {th }}$-power map on $G$ is an $H$-map. This is a significant step forward in ArkowitzCurjel and McGibbon's programme for identifying which power maps between finite $H$-spaces are $H$-maps.
\end{abstract}

\section{Introduction}

Lie groups are central objects in many areas of mathematics. Their topological properties have been used to great effect, for instance, in $K$-theory, gauge theory and the geometry of 4-manifolds. Yet many topological properties are not well understood. For example, the multiplication on a Lie group $G$ induces a power map $k: G \longrightarrow G$ for each integer $k$, defined by $k(x)=x^{k}$. When is the $k^{t h}$-power map multiplicative, at least up to homotopy? Very little is known, and this is one problem we intend to address.

Let $p$ be an odd prime. Localize all spaces and maps at $p$. Unless otherwise stated, homology will be taken with mod- $p$ coefficients. A simple, simply-connected, compact Lie group $G$ is $p$-regular if $G$ is homotopy equivalent to a product of odd dimensional spheres. More precisely, recall that $G$ is rationally homotopy equivalent to a product of spheres $G_{\mathbb{Q}} \simeq \prod_{i=1}^{l} S_{\mathbb{Q}}^{2 n_{i}-1}$. The type of $G$ is defined by the sequence $\left\{n_{1}, \ldots, n_{l}\right\}$, where $n_{1} \leqslant \cdots \leqslant n_{l}$. The group $G$ is said to be $p$-regular if there is a $p$-local homotopy equivalence $G \simeq \prod_{i=1}^{l} S^{2 n_{i}-1}$. It is classical that $G$ is $p$-regular if and only if $p \geqslant n_{l}$.

The goal of this paper is to achieve a better understanding of the homotopy theory of $p$-regular Lie groups. To set the stage, it is useful to consider a more general case. If $G$ is torsion-free, that is if $G$ has no torsion in its integral cohomology, then $H_{*}(G) \cong \Lambda(V)$ for some generating set $V$. By [T2 2$]$ the generating set $V$ can be geometrically realized: there is a space $A$ and a map $A \longrightarrow G$ which induces the inclusion of the generating set in mod- $p$ homology. Taking the adjoint, we obtain

Received December 18, 2012, revised May 31, 2013; published on August 27, 2013.

2010 Mathematics Subject Classification: Primary 55P35, Secondary 55T99.

Key words and phrases: Lie group, $p$-regular, power map.

Article available at http://intlpress.com/HHA/v15/n2/a5 and doi:10.4310/HHA.2013.v15.n2.a5

Copyright (C) 2013, International Press. Permission to copy for private use granted. 
a map $\Sigma A \stackrel{j}{\longrightarrow} B G$. This induces a fibration, up to homotopy, $Q \stackrel{\gamma}{\longrightarrow} \Sigma A \stackrel{j}{\longrightarrow} B G$. If $G$ is of low rank, for example if $G=S U(n)$ and $n \leqslant(p-1)^{2}+1$, then $\Omega j$ has a right homotopy inverse. Thus $\Omega \Sigma A \simeq G \times \Omega Q$. The motivating idea is to use this decomposition to deduce properties of $G$ from those of $\Omega \Sigma A$. This is a constructive perspective, which has proved useful in other work [GT, T2, T3].

To make the decomposition $\Omega \Sigma A \simeq G \times \Omega Q$ effective for studying $G$, one wants to know information about the space $Q$ and the map $Q \stackrel{\gamma}{\longrightarrow} \Sigma A$. For example, what is the homotopy type of $Q$, and what is the homotopy class of $\gamma$ ? We carry out these identifications when $G$ is $p$-regular.

To state the results, we need some information on homotopy commutativity (when localized at $p$ ). McGibbon [M2] showed that $G$ is homotopy commutative in precisely one of three cases: $p>2 n_{l} ; G=G_{2}$ and $p=5$; and $G=S p(2)$ and $p=3$. In particular, if $G$ is $p$-regular then it is homotopy commutative if and only if $p>2 n_{l}$. For example, $S U(n)$ is $p$-regular if $p \geqslant n$ and homotopy commutative if $p>2 n$. We prove the following.

Theorem 1.1. Let $G$ be a p-regular simple, simply-connected, compact Lie group. Then the fibration $Q \stackrel{\gamma}{\longrightarrow} \Sigma A \stackrel{j}{\longrightarrow} B G$ has the following properties:

(a) there is a homotopy equivalence $e: \Sigma R \longrightarrow Q$ where $R$ is a wedge of spheres;

(b) if $G$ is homotopy commutative then the equivalence e in part (a) can be chosen so that $\gamma \circ e$ is a sum of iterated Whitehead products;

(c) if $G$ is not homotopy commutative then the equivalence $e$ in part (a) can be chosen so that $\gamma \circ e$ is a sum of: (i) iterated Whitehead products and (ii) maps of the form $w+a$ where $w$ is an iterated Whitehead product and a depends on the stable map $\alpha_{1}$.

More precise descriptions of the map $\gamma$ in parts (b) and (c) are given in Propositions 2.8 and 2.12 respectively. In particular, the identification in part (c) relies heavily on recent work of Kaji and Kishimoto $[\mathbf{K K}]$ on the homotopy nilpotency class of $G$.

The structural properties in Theorem 1.1 are very useful for proving properties of $G$. One application occurs in [KKT], where Theorem 1.1 is used in the commutative case to help show that a certain gauge group associated to $G$ is also homotopy commutative. In this paper we present a second application.

Theorem 1.2. Let $G$ be a p-regular simple, simply-connected, compact Lie group, where $p \geqslant 5$. Then the $p^{\text {th }}$-power map on $G$ is an H-map.

An interesting immediate consequence of Theorem 1.2 is the following.

Corollary 1.3. Let $Y$ be a pointed $C W$-complex and let $p \geqslant 5$. Then the group of $p$-local homotopy classes of maps $[Y, G]$ has the property that multiplication by $p$ is a group homomorphism.

At the prime 3, McGibbon [M1] showed that the $3^{r d}$-power map on $S U(2)$ is an $H$ map. Since $S U(2)$ is homeomorphic to $S p(1)$ and $\operatorname{Spin}(3)$, these cases are covered too. The one remaining 3-regular simple, simply-connected compact Lie groups is $S U(3)$. It is not clear what happens in this case. In particular, the homotopy nilpotency class 
of $S U(3)$ is 3, matching the prime, and this leads to an obstruction for the argument in Proposition 4.4.

Theorem 1.2 is a special case of a general problem, which can be stated integrally or $p$-locally. Let $X$ be a finite, connected, homotopy associative $H$-space. For an integer $k$, let $k: X \longrightarrow X$ be the $k^{t h}$-power map, defined by $k(x)=x^{k}$. It is natural to ask whether $k$ is an $H$-map. McGibbon [M1], following earlier work of Arkowitz and Curjel [AC], showed that integrally there is a positive integer $N$ such that the $k^{\text {th }}$ power map is an $H$-map if and only if $k(k-1) \equiv 0 \bmod N$. The number $N$ measures the lack of homotopy commutativity in $X$; in particular, $N$ is a multiple of the order of the Samelson product of the identity map on $X$ with itself. However, the number $N$ is known in only a few special cases when the rank of $X$ is 1 or 2 (see [AC, M1]). As a non-local example, $N=24$ for $X=S^{3}$, where the multiplication is from regarding $S^{3}$ as the unit quaternions. As a local example, for $X=S U(3)$ with the standard loop multiplication, the $p$-component of $N$ is 5 if $p=5$ and 0 if $p>5$.

In [M1], McGibbon went on to relate the number $N$ to certain commutativity properties of $X$. He showed that if $Y$ is a finite, pointed $C W$-complex and $H=[Y, X]$, the following hold integrally:

(i) if $k(k-1) \equiv 0 \bmod N$ then $(a b)^{k}=a^{k} b^{k}$ for every $a, b \in H$;

(ii) for every $h \in H, h^{N}$ is in the centre of $H$;

(iii) the exponent of the commutator subgroup $[H, H]$ divides $N$.

Let us consider the number $N$ and properties (i) to (iii) in our $p$-local context. Let $N_{p}$ be the $p$-component of the number $N$. Assume that $G$ is a $p$-regular, simple, simply-connected, compact Lie group which is not homotopy commutative when localized at $p$. The non-homotopy commutativity of $G$ implies that the Samelson product of the identity map on $G$ with itself has order $p^{t}$ for some $t \geqslant 1$. As $N_{p}$ is a multiple of the order of this Samelson product, we see that $p$ divides $N_{p}$. On the other hand, by Theorem 1.2, the $p^{t h}$-power map on $G$ is an $H$-map, so McGibbon's result implies that $p(p-1) \equiv 0 \bmod N_{p}$. Since $p-1$ is a unit in the $p$-local integers, this implies that $p \equiv 0 \bmod N_{p}$. Thus $N_{p}$ divides $p$, and hence $N_{p}=p$. Since Theorem 1.2 holds for arbitrarily high ranks as $p$ increases, this calculation of $N_{p}$ is a substantial step forward compared to McGibbon's work in determining families of examples where the value of $N$ is known.

The author would like to thank the referee for a careful reading of the paper and for pointing out an error at the prime 3 in the original manuscript.

\section{A homotopy fibration related to $G$}

Let $G$ be a $p$-regular simple, simply-connected, compact Lie group with type $\left\{n_{1}, \ldots, n_{l}\right\}$. Localize at $p$, so there is a homotopy equivalence $G \simeq \prod_{i=1}^{l} S^{2 n_{i}-1}$. Let

$A=\bigvee_{i=1}^{l} S^{2 n_{i}-1}$. Including the wedge into product, we obtain a map $i: A \longrightarrow G$. Since $G$ is a loop space, $G \simeq \Omega B G$. Let $j: \Sigma A \longrightarrow B G$ be the adjoint of $i$. Then we obtain a homotopy fibration sequence

$$
\Omega Q \stackrel{\Omega \gamma}{\longrightarrow} \Omega \Sigma A \stackrel{\Omega j}{\longrightarrow} G \stackrel{\partial}{\longrightarrow} Q \stackrel{\gamma}{\longrightarrow} \Sigma A \stackrel{j}{\longrightarrow} B G
$$


which defines the space $Q$ and the maps $\gamma$ and $\partial$. In this section we will identify the homotopy type of $Q$, describe the map $\gamma$ and prove Theorem 1.1.

It will be useful to first record a decomposition of $\Omega \Sigma A$.

Lemma 2.1. In the homotopy fibration $\Omega Q \stackrel{\Omega \gamma}{\longrightarrow} \Omega \Sigma A \stackrel{\Omega j}{\longrightarrow} G$, the map $\Omega j$ has a right homotopy inverse, and consequently there is a homotopy decomposition

$$
\Omega \Sigma A \simeq G \times \Omega Q .
$$

Proof. By definition, $j$ is the adjoint of the composite

$$
\bigvee_{i=1}^{l} S^{2 n_{i}-1} \stackrel{I}{\longrightarrow} \prod_{i=1}^{l} S^{2 n_{i}-1} \stackrel{\simeq}{\longrightarrow} G
$$

where $I$ is the inclusion of the wedge into the product. By the Hilton-Milnor Theorem, $\Omega I$ has a right homotopy inverse. Therefore the homotopy equivalence $G \simeq$ $\prod_{i=1}^{l} S^{2 n_{i}-1}$ implies that $\Omega j$ has a right homotopy inverse. The homotopy decomposition for $\Omega \Sigma A$ follows immediately.

Consider next how the homotopy fibration $\Omega Q \stackrel{\Omega \gamma}{\longrightarrow} \Omega \Sigma A \stackrel{\Omega j}{\longrightarrow} G$ behaves in homology. Throughout we write $H_{*}(X)$ for homology with mod- $p$ coefficients. By the BottSamelson Theorem, there is an algebra isomorphism $H_{*}(\Omega \Sigma A) \cong T\left(\widetilde{H}_{*}(A)\right)$, where the right side is the tensor algebra generated by the vector space $\widetilde{H}_{*}(A)$. We also have $H_{*}(G) \cong \Lambda\left(\widetilde{H}_{*}(A)\right)$, where the right side is the exterior algebra generated by the vector space $\widetilde{H}_{*}(A)$. The definition of $j$ as the adjoint of $i$ implies that the composite $A \stackrel{E}{\longrightarrow} \Omega \Sigma A \stackrel{\Omega j}{\longrightarrow} G$ is homotopic to $i$, where $E$ is the canonical suspension. Observe that $i_{*}$ is the inclusion of the generating set into $H_{*}(G)$, and $(\Omega j)_{*}$ is a multiplicative extension of $i_{*}$. Therefore $(\Omega j)_{*}$ is the abelianization of the tensor algebra.

To describe $H_{*}(\Omega Q)$ and $(\Omega \gamma)_{*}$, it is useful to reinterpret the abelianization map $a: T\left(\widetilde{H}_{*}(A)\right) \longrightarrow \Lambda\left(\widetilde{H}_{*}(A)\right)$. As in $[\mathbf{C M N}]$, there is an isomorphism $T\left(\widetilde{H}_{*}(A)\right) \cong$ $U L\left\langle\widetilde{H}_{*}(A)\right\rangle$, where the right side is the universal enveloping algebra of the free Lie algebra generated by the elements of $\widetilde{H}_{*}(A)$. Also, there is an isomorphism $\Lambda\left(\widetilde{H}_{*}(A)\right) \cong U L_{a b}\left\langle\widetilde{H}_{*}(A)\right\rangle$, where the right side is the universal enveloping algebra of the free abelian Lie algebra (characterized by having its bracket identically zero) generated by the elements of $\widetilde{H}_{*}(A)$. The abelianization map $a$ is then identified with $U \pi$, where $\pi: L\left\langle\widetilde{H}_{*}(A)\right\rangle \longrightarrow L_{a b}\left\langle\widetilde{H}_{*}(A)\right\rangle$ is the Lie algebra map that sends any bracket in the domain to zero. Let $L=L\left\langle\widetilde{H}_{*}(A)\right\rangle$, and $L_{a b}=L_{a b}\left\langle\widetilde{H}_{*}(A)\right\rangle$. As in [CMN], there is a short exact sequence of Lie algebras

$$
0 \longrightarrow[L, L] \stackrel{\epsilon}{\longrightarrow} L \stackrel{\pi}{\longrightarrow} L_{a b} \longrightarrow 0
$$

where $[L, L]$ is the free Lie algebra generated by the brackets in $L$. This short exact sequence of Lie algebras induces a short exact sequence of Hopf algebras

$$
U[L, L] \stackrel{U \epsilon}{\longrightarrow} U L \stackrel{U \pi}{\longrightarrow} U L_{a b} .
$$

By a short exact sequence of Hopf algebras we mean that there is an isomorphism $U L \cong U[L, L] \otimes U L_{a b}$ of left $U[L, L]$-modules and right $U L_{a b}$-comodules. In our case, we obtain an isomorphism $H_{*}(\Omega \Sigma A) \cong U L_{a b} \otimes U[L, L]$ of left $U[L, L]$-modules and 
right $U L_{a b^{-}}$-comodules. On the other hand, since $(\Omega j)_{*}$ is the abelianization map, and $\Omega j$ has a right homotopy inverse by Lemma 2.1 , we obtain the following.

Lemma 2.2. There is an isomorphism of $\mathbb{Z} / p \mathbb{Z}$-vector spaces $H_{*}(\Omega Q) \cong U[L, L]$.

Note that the elements of $H_{*}(A)$ are all in odd degree. In this case, an explicit basis for $[L, L]$ was given in $[\mathbf{C N}]$. Let $\widetilde{H}_{*}(A)=\left\{u_{1}, \ldots, u_{l}\right\}$, where $\left|u_{i}\right|=2 n_{i}-1$.

Lemma 2.3. A Lie basis for $[L, L]$ is given by the elements

$$
\left[u_{i}, u_{j}\right],\left[u_{t_{1}},\left[u_{i}, u_{j}\right]\right],\left[u_{t_{2}},\left[u_{t_{1}},\left[u_{i}, u_{j}\right]\right]\right], \ldots
$$

where $1 \leqslant j \leqslant i \leqslant l$ and $1 \leqslant t_{k}<t_{k-1}<\cdots<t_{2}<t_{1}<i$. In particular, the basis elements have bracket lengths from 2 through $l+1$.

We now construct a new homotopy fibration sequence which has the same homological behavior as (1) but which may be homotopically distinct. In comparing the two we will be able to identify the homotopy type of $Q$ and the homotopy class of $\gamma$.

Observe that each element $u_{i} \in \widetilde{H}_{*}(A) \subseteq T\left(\widetilde{H}_{*}(A)\right)$ is in the image of the Hurewicz homomorphism, via the composite $\mu_{i}: S^{2 n_{i}-1} \hookrightarrow A \stackrel{E}{\longrightarrow} \Omega \Sigma A$. Thus the Lie bracket $\left[u_{i}, u_{j}\right]$ is in the image of the Hurewicz homomorphism via the Samelson product $S^{2 n_{i}+2 n_{j}-2} \cong S^{2 n_{i}-1} \wedge S^{2 n_{j}-1} \stackrel{\left\langle\mu_{i}, \mu_{j}\right\rangle}{\longrightarrow} \Omega \Sigma A$. Similarly, any iterated Lie bracket in $L \subseteq$ $U L \cong T\left(\widetilde{H}_{*}(A)\right) \cong H_{*}(\Omega \Sigma A)$ is in the image of the Hurewicz homormorphism via the corresponding iterated Samelson product of the maps $\mu_{i}$ for $1 \leqslant i \leqslant l$. In particular, every basis element of $[L, L]$ is the Hurewicz image of an iterated Samelson product.

Let $\nu_{i}: S^{2 n_{i}} \longrightarrow \Sigma A$ be the adjoint of $\mu_{i}$. The adjoint of the Samelson product $\left\langle\mu_{i}, \mu_{j}\right\rangle$ is the Whitehead product $\left[\nu_{i}, \nu_{j}\right]: S^{2 n_{i}+2 n_{j}-1} \longrightarrow \Sigma A$. Let

$$
R_{2}=\bigvee_{1 \leqslant j \leqslant i \leqslant l} S^{2 n_{i}+2 n_{j}-2}
$$

and let

$$
\lambda_{2}: \Sigma R_{2} \longrightarrow \Sigma A
$$

be the wedge sum of the Whitehead products $\left[\nu_{i}, \nu_{j}\right]$. Similarly, for $k \geqslant 3$, let $\mathcal{I}_{k}$ be an index set enumerating the basis elements of $[L, L]$ of bracket length $k$. Each $\alpha \in \mathcal{I}_{k}$ represents a bracket $\left[u_{t_{k-2}}, \ldots\left[u_{t_{1}},\left[u_{i}, u_{j}\right]\right] \ldots\right]$, which is the Hurewicz image of the iterated Samelson product $\left\langle\mu_{t_{k-2}}, \ldots\left\langle\mu_{t_{1}},\left\langle\mu_{i}, \mu_{j}\right\rangle\right\rangle \ldots\right\rangle$, whose adjoint is the corresponding iterated Whitehead product $\left[\nu_{t_{k-2}}, \ldots\left[\nu_{t_{1}},\left[\nu_{i}, \nu_{j}\right]\right] \ldots\right]$. This Whitehead product is a map $w_{k, \alpha}: S^{d_{\alpha}} \longrightarrow \Sigma A$ for $d_{\alpha}=\left(2 n_{t_{k-2}}-1\right)+\cdots+\left(2 n_{t_{1}}-1\right)+2 n_{i}+$ $2 n_{j}-1$. Let $R_{k}=\bigvee_{\alpha \in \mathcal{I}_{k}} S^{d_{\alpha}-1}$, and let

$$
\lambda_{k}: \Sigma R_{k} \longrightarrow \Sigma A
$$

be the wedge sum of the Whitehead products $w_{k, \alpha}$. Note that Lemma 2.3 implies that $\mathcal{I}_{k}=\emptyset$ for $k>l+1$. Let $R=\bigvee_{k=2}^{l+1} R_{k}$, and let

$$
\lambda: \Sigma R \longrightarrow \Sigma A
$$

be the wedge sum of the maps $\lambda_{k}$. We obtain a homotopy fibration sequence

$$
\Omega \Sigma R \stackrel{\Omega \lambda}{\longrightarrow} \Omega \Sigma A \stackrel{r}{\longrightarrow} F \stackrel{\delta}{\longrightarrow} \Sigma R \stackrel{\lambda}{\longrightarrow} \Sigma A
$$

which defines the space $F$ and the maps $\delta$ and $r$. 
Observe that the adjoint of $\lambda$ is the composite $R \stackrel{E}{\longrightarrow} \Omega \Sigma R \stackrel{\Omega \lambda}{\longrightarrow} \Omega \Sigma A$, where $E$ is the suspension map. The construction of $\lambda$ as a wedge sum of Whitehead products immediately implies the following.

Lemma 2.4. The composite $\Omega \lambda \circ E$ is a wedge sum of Samelson products, and in homology $(\Omega \lambda \circ E)_{*}$ is a monomorphism onto the vector subspace of $[L, L]$ spanned by the Lie basis of Lemma 2.3.

Since $(\Omega \lambda)_{*}$ is a multiplicative extension of $(\Omega \lambda \circ E)_{*}$, we obtain an analogue to Lemma 2.2 for (3).

Lemma 2.5. There is an isomorphism $H_{*}(\Omega \Sigma R) \cong U[L, L]$ such that $(\Omega \lambda)_{*}=U \epsilon$.

Comparing Lemmas 2.2 and 2.5, it is tempting to guess that $Q \simeq \Sigma R, \gamma \simeq \lambda$, and the two homotopy fibrations (1) and (3) are equivalent. We will show that this is true if and only if $G$ is homotopy commutative. When $G$ is not homotopy commutative we will modify $\lambda$ to give an explicit description of $\gamma$.

We begin with two general statements. First, let $f: M \longrightarrow \Omega \Sigma A$ and $g: N \longrightarrow$ $\Omega \Sigma A$ be any maps and consider the Samelson product $M \times N \stackrel{\langle f, g\rangle}{\longrightarrow} \Omega \Sigma A$. Since $\Omega \Sigma A \stackrel{\Omega i}{\longrightarrow} G$ is an $H$-map into a homotopy associative $H$-space, we have $\Omega i \circ\langle f, g\rangle \simeq$ $\langle\Omega i \circ f, \Omega i \circ g\rangle$. We record this as the following.

Lemma 2.6. Samelson products on $\Omega \Sigma A$ compose with $\Omega i$ to give Samelson products on $G$.

Second, for a space $X$, let $E: X \longrightarrow \Omega \Sigma X$ be the suspension map. The following theorem is known as the universal property of the James construction $[\mathbf{J}]$.

Theorem 2.7. Let $X$ be a path-connected space and $Y$ be a path-connected homotopy associative $H$-space. Let $f: X \longrightarrow Y$ be a map. Then $f$ extends to an $H$-map $\bar{f}: \Omega \Sigma X \longrightarrow Y$, and $\bar{f}$ is the unique $H$-map such that $\bar{f} \circ E \simeq f$.

The uniqueness statement in Theorem 2.7 implies that there is a one-to-one correspondence between the homotopy classes of maps $[A, Y]$ and the homotopy classes of $H$-maps in $[\Omega \Sigma A, Y]$. In particular, the homotopy class of any $H$-map $\Omega \Sigma A \longrightarrow Y$ is determined by its restriction to $A$.

We now specialize to the case when $G$ is homotopy commutative. Recall from [M2] that a $p$-regular group $G$ of type $\left\{n_{1}, \ldots, n_{l}\right\}$ is homotopy commutative if and only if $p>2 n_{l}$.

Proposition 2.8. If $G$ is homotopy commutative then there is a homotopy equivalence $\Sigma R \stackrel{e}{\longrightarrow} Q$ such that $\lambda \simeq \gamma \circ e$.

Proof. By Lemmas 2.4 and 2.6, the composite $R \stackrel{E}{\longrightarrow} \Omega \Sigma R \stackrel{\Omega \lambda}{\longrightarrow} \Omega \Sigma A \stackrel{\Omega i}{\longrightarrow} G$ is a wedge sum of Samelson products. Since $G$ is homotopy commutative, this composite is null homotopic. Theorem 2.7 therefore implies that $\Omega i \circ \Omega \lambda$ is null homotopic. Thus there is a lift

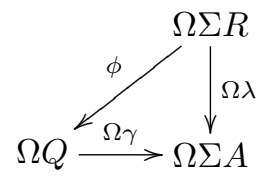

for some map $\phi$. 
We will show that this lift deloops. First observe in general that the right adjoint of a map $f: \Sigma X \longrightarrow Y$ is homotopic to the composite $X \stackrel{E}{\longrightarrow} \Omega \Sigma X \stackrel{\Omega f}{\longrightarrow} \Omega Y$, and the left adjoint of a map $g: X \longrightarrow \Omega Y$ is homotopic to the composite $\Sigma X \stackrel{\Sigma g}{\longrightarrow} \Sigma \Omega Y \stackrel{e v}{\longrightarrow}$ $Y$, where $e v$ is the canonical evaluation map. In particular, if we start with the identity map on $\Sigma X$, take the right adjoint and then take the left adjoint of that, we recover the identity map. Thus the identity map on $\Sigma X$ is homotopic to the composite $\Sigma X \stackrel{\Sigma E}{\longrightarrow} \Sigma \Omega \Sigma X \stackrel{e v}{\longrightarrow} \Sigma X$.

Now consider the diagram

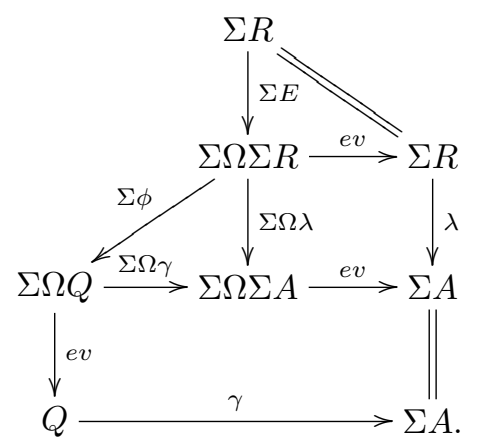

The upper triangle homotopy commutes since $e v \circ \Sigma E$ is homotopic to the identity map on $\Sigma R$. The middle left triangle homotopy commutes as it is the suspension of the previous diagram. The upper right square and bottom rectangle homotopy commute by the naturality of the evaluation for suspension loop maps. Thus the entire diagram homotopy commutes. Consider the outer perimeter of the diagram as a whole. Letting $e=e v \circ \Sigma \phi \circ \Sigma E$, we obtain a homotopy commutative diagram

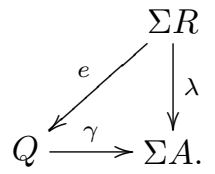

To finish the proof we need to show that $e$ is a homotopy equivalence. It suffices to show that $\Omega e$ is a homotopy equivalence, for then $\Omega e$ induces an isomorphism of homotopy groups, which implies that $e$ induces an isomorphism of homotopy groups, and so is a (weak) homotopy equivalence. Since $\Omega \Sigma R$ and $\Omega Q$ are simply-connected, to show that $\Omega e$ is a homotopy equivalence it suffices by Whitehead's Theorem to show that $(\Omega e)_{*}$ is an isomorphism.

By Lemmas 2.2 and 2.5 , we have $H_{*}(\Omega \Sigma R) \cong U[L, L] \cong H_{*}(\Omega Q)$, and $(\Omega \lambda)_{*}$ is a monomorphism. Since $(\Omega \lambda)_{*}$ is a monomorphism, the homotopy $\Omega \gamma \circ \Omega e \simeq \Omega \lambda$ implies that $(\Omega e)_{*}$ is also a monomorphism. Now $(\Omega e)_{*}$ is a monomorphism between two $\mathbb{Z} / p \mathbb{Z}$-modules with the same Euler-Poincare series. Hence $\left(\Omega e_{*}\right)$ is an isomorphism.

Corollary 2.9. If $G$ is homotopy commutative then there is a homotopy fibration $\Sigma R \stackrel{\lambda}{\longrightarrow} \Sigma A \stackrel{j}{\longrightarrow} B G$. 
Next, suppose that $G$ is not homotopy commutative. Let $x_{i}$ be the composite

$$
x_{i}: S^{2 n_{i}-1} \stackrel{\mu_{i}}{\longrightarrow} \Omega \Sigma A \stackrel{\Omega i}{\longrightarrow} G .
$$

By Lemma 2.6, $\Omega i \circ\left\langle\mu_{i}, \mu_{j}\right\rangle \simeq\left\langle x_{i}, x_{j}\right\rangle$. Let $\alpha_{1}: S^{2 p} \longrightarrow S^{3}$ represent the generator of $\pi_{2 p}\left(S^{3}\right)$, which is the least dimensional torsion homotopy group of $S^{3}$. Ambiguously, for $m \geqslant 3$, let $\alpha_{1}: S^{m+2 p-3} \longrightarrow S^{m}$ be the $(m-3)$-fold suspension of $\alpha_{1}$. Kaji and Kishimoto $[\mathbf{K K}]$ prove the following.

Lemma 2.10. Suppose that $G$ is a p-regular simple, simply-connected, compact Lie group which is not homotopy commutative. Then the following hold:

(a) at least one Samelson product $\left\langle x_{i}, x_{j}\right\rangle$ is nontrivial;

(b) if the Samelson product $\left\langle x_{i}, x_{j}\right\rangle$ is nontrivial, it is homotopic to a nonzero multiple of the composite $a_{i, j}: S^{2 n_{i}+2 n_{j}-2} \stackrel{\alpha_{1}}{\longrightarrow} S^{2 n_{i}+2 n_{j}-2 p+1} \hookrightarrow \prod_{i=1}^{l} S^{2 n_{i}-1} \simeq G$;

(c) the Samelson product $\left\langle x_{t},\left\langle x_{i}, x_{j}\right\rangle\right\rangle$ is nontrivial only if

$$
2 n_{t}+2 n_{i}+2 n_{j}=4 p
$$

(d) when $\left\langle x_{t},\left\langle x_{i}, x_{j}\right\rangle\right\rangle$ is nontrivial, it is homotopic to a nonzero multiple of the composite $S^{4 p-3} \stackrel{\alpha_{1}}{\longrightarrow} S^{2 p} \stackrel{\alpha_{1}}{\longrightarrow} S^{3}$.

Lemma 2.10 is the main technical ingredient in $[\mathbf{K K}]$ for proving the nilpotency result in Theorem 2.11. To state this, we require a definition. In general, for an $\mathrm{H}$ space $X$ with a homotopy inverse, let $c: X \times X \longrightarrow X$ be the commutator, defined pointwise by $c(x, y)=x y x^{-1} y^{-1}$. Let $c_{0}$ be the identity map on $X$, and for $k \geqslant 1$, let $c_{k}: X^{k+1} \longrightarrow X$ be the iterated commutator map

$$
c_{k}=c \circ(1 \times c) \circ \cdots \circ(1 \times \cdots \times 1 \times c)
$$

where $X^{k+1}$ is the product of $k+1$ copies of $X$. We say that $\operatorname{nil}(X)=m$ if $c_{m}$ is null homotopic and $c_{m-1}$ is not null homotopic. Observe that $X$ is homotopy commutative if and only if $\operatorname{nil}(X)=1$.

Theorem 2.11. Let $G$ be a p-regular simple, simply-connected, compact Lie group of type $\left\{n_{1}, \ldots, n_{l}\right\}$. Then the following hold:

(a) $\operatorname{nil}(G)=2$ if $\frac{3}{2} n_{l}<p<2 n_{l}$;

(b) $\operatorname{nil}(G)=3$ if $n_{l} \leqslant p \leqslant \frac{3}{2} n_{l}$ except for $(G, p)$ equal to one of $\left(F_{4}, 17\right),\left(E_{6}, 17\right)$, $\left(E_{8}, 41\right)$ or $\left(E_{8}, 43\right)$;

(c) in the exceptional cases in part (b), $\operatorname{nil}(G)=2$.

Part (a) of Lemma 2.10 is a converse to Proposition 2.8. By the definition of $\lambda$, each Samelson product $\left\langle\mu_{i}, \mu_{j}\right\rangle$ factors through $\Omega \lambda$. Thus as $\left\langle x_{i}, x_{j}\right\rangle=\Omega i \circ\left\langle\mu_{i}, \mu_{j}\right\rangle$ is nontrivial, the composite $\Omega \Sigma R \stackrel{\Omega \lambda}{\longrightarrow} \Omega \Sigma A \stackrel{\Omega i}{\longrightarrow} G$ is nontrivial. Hence there is no lift of $\lambda$ through $\gamma$.

However, Lemma 2.10 (b) together with the homotopy nilpotency class of $G$ lets us modify $\lambda$ to obtain a lift through $\gamma$. The next several paragraphs describe this modification explicitly, culminating in Proposition 2.12. By definition, $R=\bigvee_{k=2}^{l+1} R_{k}$ and $\lambda=\bigvee_{k=2}^{l+1} \lambda_{k}$, where $R_{k}$ is a wedge of spheres and $\Sigma R_{k} \stackrel{\lambda_{k}}{\longrightarrow} \Sigma A$ is a wedge sum of Whitehead products which are in one-to-one correspondence with the Lie basis 
elements of $[L, L]$ of length $k$. Taking the adjoint, the composite $R_{k} \stackrel{E}{\longrightarrow} \Omega \Sigma R_{k} \stackrel{\Omega \lambda_{k}}{\longrightarrow}$ $\Omega \Sigma A$ is a wedge sum of Samelson products whose Hurewicz images are the Lie basis elements of $[L, L]$ of length $k$. Since $G$ is of nilpotence class at most 3 , the composite $R_{k} \stackrel{E}{\longrightarrow} \Omega \Sigma R_{k} \stackrel{\Omega \lambda_{k}}{\longrightarrow} \Omega \Sigma A \stackrel{\Omega i}{\longrightarrow} G$ is null homotopic if $k>3$. Thus for each $k \geqslant 4$ there is a lift

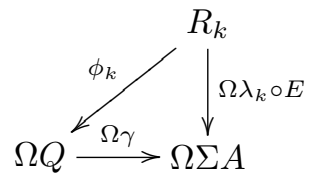

for some map $\phi_{k}$.

Next, consider the composite $R_{2} \stackrel{E}{\longrightarrow} \Omega \Sigma R_{2} \stackrel{\Omega \lambda_{2}}{\longrightarrow} \Omega \Sigma A$. By definition, $R_{2}$ is a wedge of spheres and $\Omega \lambda_{2} \circ E$ is a wedge sum of the Samelson products $\left\langle\mu_{i}, \mu_{j}\right\rangle$ for $i \geqslant j$. So the composite $R_{2} \stackrel{\Omega \lambda_{2} \circ E}{\longrightarrow} \Omega \Sigma A \stackrel{\Omega i}{\longrightarrow} G$ is a wedge sum of the Samelson products $\left\langle x_{i}, x_{j}\right\rangle$ for $i \geqslant j$. Some of these Samelson products may be null homotopic, and some may not; by Lemma 2.10 (a), at least one is not. Let $R_{2,1}$ be the subwedge of spheres in $R_{2}$ whose corresponding Samelson products $\left\langle x_{i}, x_{j}\right\rangle$ are null homotopic, and let $R_{2,2}$ be the subwedge of spheres in $R_{2}$ whose corresponding Samelson products $\left\langle x_{i}, x_{j}\right\rangle$ are nontrivial. Then $R_{2}=R_{2,1} \vee R_{2,2}$ and the restriction of $R_{2} \stackrel{\Omega \lambda_{2} \circ E}{\longrightarrow} \Omega \Sigma A \stackrel{\Omega i}{\longrightarrow} G$ to $R_{2,1}$ is null homotopic. We now modify the restriction of $\Omega \lambda_{2} \circ E$ to $R_{2,2}$ so that it also composes trivially with $\Omega i$. Suppose that the Samelson product $\left\langle x_{i}, x_{j}\right\rangle$ is nontrivial. Let $b_{i, j}$ be the composite

$$
b_{i, j}: S^{2 n_{i}+2 n_{j}-2} \stackrel{\alpha_{1}}{\longrightarrow} S^{2 n_{i}+2 n_{j}-2 p+1} \hookrightarrow \bigvee_{i=1}^{l} S^{2 n_{i}-1} \simeq A
$$

Observe that the composite $S^{2 n_{i}+2 n_{j}-2} \stackrel{b_{i, j}}{\longrightarrow} A \stackrel{E}{\longrightarrow} \Omega \Sigma A \stackrel{\Omega i}{\longrightarrow} G$ is homotopic to a nonzero multiple of the composite $a_{i, j}$ in Lemma 2.10. Let $d_{i, j}=\left\langle\mu_{i}, \mu_{j}\right\rangle-b_{i, j}$. Since $b_{i, j}$ and $\left\langle\mu_{i}, \mu_{j}\right\rangle$ factor through $a_{i, j}$ and $\left\langle x_{i}, x_{j}\right\rangle$ respectively, Lemma 2.10 (b) implies that the composite $\Omega i \circ d_{i, j}$ is null homotopic. Thus the difference $d_{i, j}$ lifts through $\Omega \gamma$. Collecting all these differences, one for each wedge summand of $R_{2,2}$, we obtain a map $D_{2}: R_{2,2} \longrightarrow \Omega \Sigma A$ with the property that $\Omega i \circ D_{2}$ is null homotopic. Define $\theta_{2}: R_{2}=R_{2,1} \vee R_{2,2} \longrightarrow \Omega \Sigma A$ as the wedge sum of $\lambda_{2} \circ E$ restricted to $R_{2,1}$ and $D_{2}$ on $R_{2,2}$. Then $\Omega i \circ \theta_{2}$ is null homotopic, so we obtain a lift

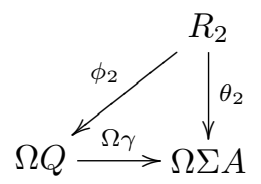

for some map $\phi_{2}$.

Similarly, we write $R_{3}=R_{3,1} \vee R_{3,2}$ where $R_{3,1}$ ( $R_{3,2}$ respectively) is the subwedge of spheres in $R_{3}$ whose corresponding Samelson products $\left\langle\mu_{t_{1}},\left\langle\mu_{i}, \mu_{j}\right\rangle\right\rangle$ compose trivially (nontrivially) with $\Omega \Sigma A \stackrel{\Omega i}{\longrightarrow} G$. If the homotopy nilpotency class of $G$ is 2 , then $R_{3,2}=*$ and $R_{3}=R_{3,1}$. If the homotopy nilpotency class of $G$ is 3, then Lemma 2.10 (c) describes the nontrivial Samelson products $\left\langle x_{t},\left\langle x_{i}, x_{j}\right\rangle\right\rangle$. Any such 
Samelson product has $2 n_{t}+2 n_{i}+2 n_{j}=4 p$ and is homotopic to a nonzero multiple of the composite $S^{4 p-3} \stackrel{\alpha_{1}}{\longrightarrow} S^{2 p} \stackrel{\alpha_{1}}{\longrightarrow} S^{3}$. Observe that by definition, $\left\langle x_{t},\left\langle x_{i}, x_{j}\right\rangle\right\rangle \simeq$ $\Omega i \circ\left\langle\mu_{t},\left\langle\mu_{i}, \mu_{j}\right\rangle\right\rangle$, and $\alpha_{1} \circ \alpha_{1}$ factors as $\Omega i \circ \bar{\alpha}$ where $\bar{\alpha}$ is the composite $\bar{\alpha}: S^{4 p-3} \stackrel{\alpha_{1}}{\longrightarrow}$ $S^{2 p} \stackrel{\alpha_{1}}{\longrightarrow} S^{3} \hookrightarrow \bigvee_{i=1}^{l} S^{2 n_{i}-1}=A \stackrel{E}{\longrightarrow} \Omega \Sigma A$. In this case, let $d_{t, i, j}=\left\langle\mu_{t},\left\langle\mu_{i}, \mu_{j}\right\rangle\right\rangle-\bar{\alpha}$. Then $\Omega i \circ d_{t, i, j}$ is null homotopic, so the difference $d_{t, i, j}$ lifts through $\Omega \gamma$. Collecting all these differences, one for each wedge summand of $R_{3,2}$, we obtain a map $D_{3}: R_{3,2} \longrightarrow \Omega \Sigma A$ with the property that $\Omega i \circ D_{3}$ is null homotopic. Define $\theta_{3}: R_{3}=R_{3,1} \vee R_{3,2} \longrightarrow \Omega \Sigma A$ as the wedge sum of $\lambda_{3} \circ E$ restricted to $R_{3,1}$ and $D_{3}$ on $R_{3,2}$. Then $\Omega i \circ \theta_{3}$ is null homotopic, so we obtain a lift

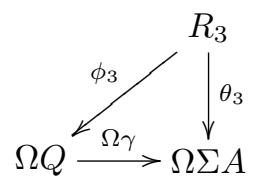

for some map $\phi_{3}$.

Let $\theta: R \longrightarrow \Omega \Sigma A$ be the wedge sum of the maps $\theta_{2}, \theta_{3}$ and $\Omega \lambda_{k} \circ E$ for $4 \leqslant k \leqslant$ $l+1$, and let $\phi: R \longrightarrow \Omega Q$ be the wedge sums of the maps $\phi_{k}$ for $2 \leqslant k \leqslant l+1$. Then there is a homotopy commutative diagram

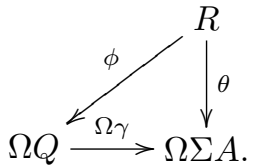

Taking adjoints, let $\vartheta=e v \circ \Sigma \theta$ and $\varphi=e v \circ \Sigma \phi$. Then there is a homotopy commutative diagram

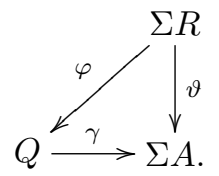

Proposition 2.12. The map $\Sigma R \stackrel{\varphi}{\longrightarrow} Q$ is a homotopy equivalence.

Proof. It suffices to show that $(\Omega \varphi)_{*}$ is an isomorphism. For then, as $\Omega \Sigma R$ and $\Omega Q$ are simply-connected, Whitehead's theorem implies that $\Omega \varphi$ is a homotopy equivalence. Thus $\Omega \varphi$ induces an isomorphism on homotopy groups, implying that $\varphi$ induces an isomorphism on homotopy groups, and hence $\varphi$ is a (weak) homotopy equivalence.

Consider the loops on (4). By Lemma $2.2, H_{*}(\Omega Q) \cong U[L, L]$ as vector spaces, and by Lemma $2.5, H_{*}(\Omega \Sigma R) \cong U[L, L]$ as algebras. Suppose that $(\Omega \vartheta)_{*}$ is a monomorphism. Then the homotopy commutativity of (4) implies that $(\Omega \varphi)_{*}$ is also a monomorphism. Thus $(\Omega \varphi)_{*}$ is a monomorphism between two $\mathbb{Z} / p \mathbb{Z}$-vector spaces with the same Euler-Poincaré series and so is an isomorphism.

It remains to show that $(\Omega \vartheta)_{*}$ is a monomorphism. Since $(\Omega \vartheta)_{*}$ is an algebra map whose domain is the tensor algebra $H_{*}(\Omega \Sigma R) \cong T\left(\widetilde{H}_{*}(R)\right)$, the image of $(\Omega \vartheta)_{*}$ is determined by its restriction to the generating set $\widetilde{H}_{*}(R)$. On the level of spaces, this corresponds to finding the image in homology of the composite $R \stackrel{E}{\longrightarrow} \Omega \Sigma R \stackrel{\Omega \vartheta}{\longrightarrow} \Omega \Sigma A$. 
This composite is the adjoint of $\vartheta$, which is $\theta$. We claim that $\theta_{*}=(\Omega \lambda \circ E)_{*}$. By definition, $\theta$ is the wedge sum of $\theta_{2}, \theta_{3}$ and $\Omega \lambda_{k} \circ E$ for $4 \leqslant k \leqslant l+1$, and by definition of $\lambda, \Omega \lambda \circ E$ is the wedge sum of $\Omega \lambda_{k} \circ E$ for $2 \leqslant k \leqslant l+1$. Thus it suffices to show that $\left(\theta_{2}\right)_{*}=\left(\Omega \lambda_{2} \circ E\right)_{*}$ and $\left(\theta_{3}\right)_{*}=\left(\Omega \lambda_{3} \circ E\right)_{*}$.

The map $\theta_{2}$ is the wedge sum of the restriction of $\Omega \lambda_{2} \circ E$ to $R_{2,1}$ and difference maps $d_{i, j}$ on $R_{2,2}$. Observe that the map $a_{i, j}$ in Lemma 2.10 (b) is zero in homology. Thus each difference $d_{i, j}=\left\langle\mu_{i}, \mu_{j}\right\rangle-a_{i, j}$ has the same image in homology as $\left\langle\mu_{i}, \mu_{j}\right\rangle$. Therefore $\left(\theta_{2}\right)_{*}=\left(\Omega \lambda_{2} \circ E\right)_{*}$. A similar argument shows that $\left(\theta_{3}\right)_{*}=\left(\Omega \lambda_{3} \circ E\right)_{*}$, and this completes the proof.

The homotopy equivalence of Proposition 2.12 combined with the homotopy commutative diagram (4) immediately implies the following.

Corollary 2.13. There is a homotopy fibration

$$
\Sigma R \stackrel{\vartheta}{\longrightarrow} \Sigma A \stackrel{j}{\longrightarrow} B G .
$$

Collecting the results so far, we obtain the goal of this section.

Proof of Theorem 1.1. Part (a) follows from the fact that $R$ is a wedge of spheres in Corollaries 2.9 and 2.13. Part (b) now follows from the definition of $\lambda$ and Proposition 2.8, while part (c) follows from the definition of $\vartheta$ and Proposition 2.12.

Ideally, one would like to know precisely when the Samelson products $\left\langle x_{i}, x_{j}\right\rangle$ and $\left\langle x_{t},\left\langle x_{i}, x_{j}\right\rangle\right\rangle$ are nontrivial. Then $R_{2,1}, R_{2,2}, R_{3,1}$ and $R_{3,2}$ can be identified, and the map $\varphi$ is completely determined. However, this is known at present only when $G$ is homotopy commutative, in which case all the $\left\langle x_{i}, x_{j}\right\rangle$ are trivial, or when $G$ is one of $S U(n), S p(n)$ or $\operatorname{Spin}(2 n+1)$ - to be described momentarily. When $G$ is $\operatorname{Spin}(2 n)$ or an exceptional Lie group, and not homotopy commutative, not enough is known about the Samelson products $\left\langle x_{i}, x_{j}\right\rangle$. Recently, $[\mathbf{H K}]$ and $[\mathbf{K K}]$ have given examples of nontrivial Samelson products in certain cases, but a good deal needs to be done before a complete answer is obtained.

We consider the case of $G=S U(n)$ in detail. If $p>2 n$ then by [M2], $S U(n)$ is homotopy commutative, in which case Lemma 2.8 implies that there is a homotopy fibration $\Sigma R \stackrel{\lambda}{\longrightarrow} \Sigma A \stackrel{i}{\longrightarrow} B S U(n)$ where $\lambda$ is given entirely by Whitehead products.

If $p<2 n$ then $S U(n)$ is not homotopy commutative. Bott [Bo] showed that if $i+j>n$, the order of the Samelson product $\left\langle x_{i}, x_{j}\right\rangle$ is a non-zero multiple of

$$
\nu_{p}\left(\frac{(i+j-1) !}{(i-1) !(j-1) !}\right)
$$

where $\nu_{p}\left(p^{t} q\right)=p^{t}$ if $(p, q)=1$. In our case, $S U(n)$ is $p$-regular so $n \leqslant p$. Thus $i \leqslant p$ and $j \leqslant p$ so $\nu_{p}((i-1) !(j-1) !)=1$. Therefore (5) implies that $\left\langle x_{i}, x_{j}\right\rangle$ is nontrivial if and only if $i+j-1 \geqslant p$. Thus $R_{2,1}=\bigvee_{i+j-1<p} S^{2 n_{i}+2 n_{j}-2}$ and $R_{2,2}=$ $\bigvee_{i+j-1 \geqslant p} S^{2 n_{i}+2 n_{j}-2}$.

If $\frac{3}{2} n<p<2 n$ then $\operatorname{nil}(S U(n))=2[\mathbf{K K}]$, implying that $R_{3}=R_{3,1}$ and $\vartheta_{3}=$ $\lambda_{3}$. This completes the description of $\Sigma R$ and $\vartheta$ in this case. If $n \leqslant p \leqslant \frac{3}{2} n$ then $\operatorname{nil}(S U(n))=3[\mathbf{K K}]$. By Lemma 2.10 (c), the Samelson product $\left\langle x_{t},\left\langle x_{i}, x_{j}\right\rangle\right\rangle$ is nontrivial if and only if $2 n_{t}+2 n_{i}+2 n_{j}=4 p$ and $\left\langle x_{t},\left\langle x_{i}, x_{j}\right\rangle\right\rangle$ is homotopic to the composite $S^{4 p-3} \stackrel{\alpha_{1}}{\longrightarrow} S^{2 p} \stackrel{\alpha_{1}}{\longrightarrow} S^{3}$. Thus $R_{3,2}=\bigvee_{\mathcal{I}} S^{4 p}$ where $\mathcal{I}$ runs over all triples 
$(t, i, j)$ such that $\left\langle x_{t},\left\langle x_{i}, x_{j}\right\rangle\right\rangle \simeq \alpha_{1} \circ \alpha_{1}$, and $R_{3,1}$ is the complement of $R_{3,2}$ in $R_{3}$. This completes the description of $\Sigma R$ and $\vartheta$ in this case.

A similar analysis in the case when $G=S p(n)$ can be made using the following: (i) if $4 p>n$ then $S p(n)$ is homotopy commutative [M2]; (ii) if $2 p<n<4 p$ then [Bo] states that for $i+j-1>n$ the order of the Samelson product $\left\langle x_{i}, x_{j}\right\rangle$ is a non-zero multiple of $\nu_{p}\left(\frac{(2 i+2 j-1) !}{(2 i-1) !(2 j-1) !}\right)$; (iii) if $3 n<p<4 n$ then nil $(S p(n))=2[\mathbf{K K}]$; and (iv) if $2 n<p<3 n$ then $\operatorname{nil}(S p(n))=3[\mathbf{K K}]$.

In the case when $G=\operatorname{Spin}(2 n+1)$, by $[\mathbf{F}]$ there is a homotopy equivalence of loop spaces (localized at an odd prime) $\operatorname{Spin}(2 n+1) \simeq S p(n)$, so the description of $\Sigma R$ and $\vartheta$ in this case is identical to that when $G=S p(n)$.

\section{Constructing certain $H$-maps}

In this section we give conditions for the existence and uniqueness of $H$-maps between certain homotopy associative $H$-spaces. The ideas are similar to those in $[\mathbf{G r}$, G1, G2, T1, T2], where the $H$-spaces considered are also homotopy commutative, and the aim is to prove a certain "universal" property. Here, we drop the commutativity hypothesis and do not attempt anything as grand as a universal statement. It may be useful to recall Theorem 2.7, as it will be used repeatedly.

To set up, let $Y$ be a homotopy associative $H$-space, and suppose that there is a space $X$ and a map $i: X \longrightarrow Y$ such that $H_{*}(Y) \cong \Lambda\left(\widetilde{H}_{*}(X)\right)$, with $i_{*}$ inducing the inclusion of the generating set. By Theorem 2.7, there is an $H$-map $\bar{i}: \Omega \Sigma X \longrightarrow$ $Y$ such that $\bar{i} \circ E \simeq i$. Notice that $\bar{i}_{*}$ is the multiplicative extension of $i_{*}$, so $\bar{i}_{*}$ is the abelianization map, $H_{*}(\Omega \Sigma A) \cong T\left(\widetilde{H}_{*}(A)\right) \stackrel{a b}{\longrightarrow} \Lambda\left(\widetilde{H}_{*}(A)\right) \cong H_{*}(Y)$. Define the space $F$ and the map $h$ by the homotopy fibration

$$
F \stackrel{h}{\longrightarrow} \Omega \Sigma X \stackrel{\bar{i}}{\longrightarrow} Y \text {. }
$$

Suppose that $\bar{i}$ has a right homotopy inverse $s: Y \longrightarrow \Omega \Sigma X$. Then the composite

$$
e: Y \times F \stackrel{s \times h}{\longrightarrow} \Omega \Sigma X \times \Omega \Sigma X \stackrel{\mu}{\longrightarrow} \Omega \Sigma X
$$

is a homotopy equivalence, where $\mu$ is the loop multiplication.

Now suppose that $Z$ is a homotopy associative $H$-space, and let $f: X \longrightarrow Z$ be any map. By Theorem 2.7, $f$ extends to an $H$-map $\bar{f}: \Omega \Sigma X \longrightarrow Z$ such that $\bar{f} \circ E \simeq f$, and $f$ is the unique $H$-map with this property. Let $g$ be the composite $Y \stackrel{s}{\longrightarrow} \Omega \Sigma X \stackrel{\bar{f}}{\longrightarrow} Z$.

Lemma 3.1. Suppose that the composite $F \stackrel{h}{\longrightarrow} \Omega \Sigma X \stackrel{\bar{f}}{\longrightarrow} Z$ is null homotopic. Then there is a homotopy commutative diagram

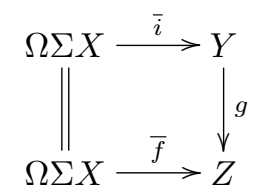

where $g$ can be chosen to be an H-map. 
Proof. Consider the diagram

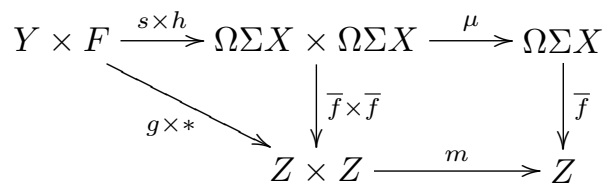

where $m$ is the multiplication on $Z$. The left triangle homotopy commutes by the definition of $g$ and the hypothesis that $\bar{f} \circ h$ is null homotopic. The square homotopy commutes since $\bar{f}$ is an $H$-map. The composite along the top row is the definition of the homotopy equivalence $e$. Thus the diagram implies that $\bar{f} \circ e \simeq g \circ \pi_{1}$, where $\pi_{1}$ is the projection onto $Y$. Let $t: \Omega \Sigma X \longrightarrow Y \times F$ be a right homotopy inverse of $e$, and precompose (6) with $t$. Then we obtain a homotopy commutative diagram

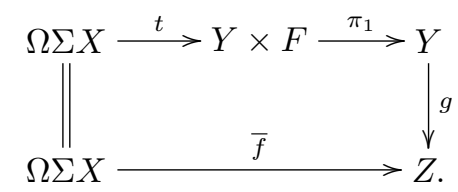

Next, we identify the homotopy class $\pi_{1} \circ t$. In (6), take $Z=Y$ and $\bar{f}=\bar{i}$. In this case, $g$ is homotopic to the identity map on $Y$. Therefore (7) implies that $\pi_{1} \circ t \simeq \bar{i}$. Thus (7) can be rewritten as a homotopy commutative diagram

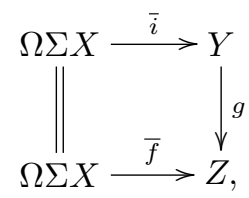

which is the diagram asserted by the lemma.

It remains to show that $g$ is an $H$-map. Consider the diagram

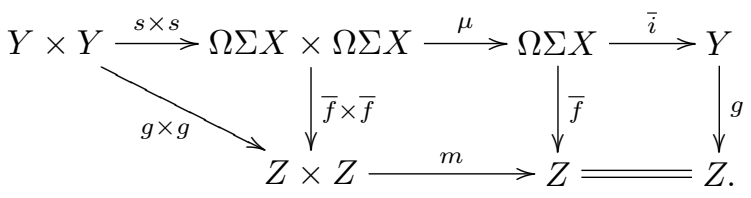

The left triangle homotopy commutes by the definition of $g$, the middle square homotopy commutes since $\bar{f}$ is an $H$-map, and we have just seen that the right square homotopy commutes. Let $m_{Y}$ be the given $H$-structure on $Y$, and let $m_{Y}^{\prime}=\bar{i} \circ \mu \circ(s \times s)$ be the $H$-structure on $Y$ determined by the composite along the top row of (8). The homotopy commutativity of (8) implies that $g$ is an $H$-map with respect to the $H$ structure $m_{Y}^{\prime}$ on $Y$. We wish to show that $g$ is an $H$-map with respect to $m_{Y}$. We do so by showing that $m_{Y} \simeq m_{Y}^{\prime}$. In (8) take $Z=Y, \bar{f}=\bar{i}$, and $m=m_{Y}$. In this case, $g$ is the identity map on $Y$. Therefore (8) implies that $m_{Y}^{\prime} \simeq m_{Y}$, as desired.

Although not strictly necessary in what follows, we now improve Lemma 3.1 by showing that the $H$-map $g$ extends the initial map $X \stackrel{f}{\longrightarrow} Z$, and has a uniqueness property. 
Proposition 3.2. Let $f: X \longrightarrow Z$ be a map such that the composite $F \stackrel{h}{\longrightarrow} \Omega \Sigma X \stackrel{\bar{f}}{\longrightarrow}$ $Z$ is null homotopic. Then $f$ extends to an $H$-map

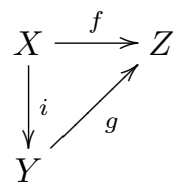

and $g$ is the unique $H$-map such that $g \circ i \simeq f$.

Proof. Consider the diagram

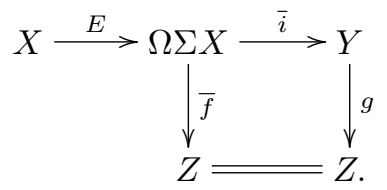

By Lemma 3.1, the square homotopy commutes and $g$ is an $H$-map. By the definitions of $\bar{f}$ and $\bar{i}$, we have $\bar{f} \circ E \simeq f$ and $\bar{i} \circ E \simeq i$. Thus this diagram implies that $g \circ i \simeq f$, proving the homotopy commutativity of the diagram asserted by the lemma.

It remains to show the uniqueness property. Suppose that $g^{\prime}: Y \longrightarrow Z$ is another $H$-map such that $g^{\prime} \circ i \simeq f$. We need to show that $g \simeq g^{\prime}$. Consider the composites $\psi: \Omega \Sigma X \stackrel{\bar{i}}{\longrightarrow} Y \stackrel{g}{\longrightarrow} Z$ and $\psi^{\prime}: \Omega \Sigma X \stackrel{\bar{i}}{\longrightarrow} Y \stackrel{g^{\prime}}{\longrightarrow} Z$. Both are $H$-maps as they are composites of $H$-maps. Observe that $\psi \circ E \simeq g \circ \bar{i} \circ E \simeq g \circ i \simeq f$, and similarly $\psi^{\prime} \circ E \simeq f$. Thus $\psi$ and $\psi^{\prime}$ are $H$-maps extending $f$. The uniqueness property of Theorem 2.7 implies that $\psi \simeq \psi^{\prime}$. Since $\bar{i}$ has a right homotopy inverse $s$, we have a string of homotopies

$$
g \simeq g \circ \bar{i} \circ s \simeq \psi \circ s \simeq \psi^{\prime} \circ s \simeq g^{\prime} \circ \bar{i} \circ s \simeq g^{\prime} .
$$

\section{The $p^{\text {th }}$-power map on $G$}

If $X$ is an $H$-space, let $p: X \longrightarrow X$ be the $p^{t h}$-power map. If $Y$ is a co- $H$ space, let $\underline{p}: Y \longrightarrow Y$ be the degree $p$ map. Since $\Omega \Sigma A \stackrel{\Omega j}{\longrightarrow} G$ is a loop map, it commutes with the $p^{\text {th }}$-power map, so there is a homotopy commutative diagram

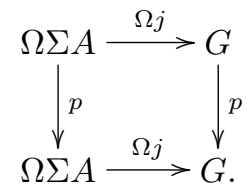

By Lemma 2.1, $\Omega j$ has a right homotopy inverse, $s: G \longrightarrow \Omega \Sigma A$. Therefore (9) implies that the $p^{t h}$-power map $G \stackrel{p}{\longrightarrow} G$ is homotopic to the composite $G \stackrel{s}{\longrightarrow} \Omega \Sigma A \stackrel{p}{\longrightarrow}$ $\Omega \Sigma A \stackrel{\Omega j}{\longrightarrow} G$.

We wish to show that the $p^{t h}$-power map on $G$ is an $H$-map. To do so, we will compare $\Omega j \circ p \circ s$ to the composite $\Omega j \circ \Omega \underline{p} \circ s$, where $\underline{p}: \Sigma A \longrightarrow \Sigma A$ is the degree $p$ map. This will bring into play the difference $\Omega \Sigma A \stackrel{p-\Omega \underline{p}}{\longrightarrow} \Omega \Sigma A$ which is controllable by means of the Hilton-Milnor Theorem. 
We begin with a preliminary observation. By definition, $A=\bigvee_{i=1}^{l} S^{2 n_{i}-1}$, so the degree $p$ map $\Sigma A \stackrel{p}{\longrightarrow} \Sigma A$ is homotopic to $\bigvee_{i=1}^{l} \Sigma S^{2 n_{i}-1} \stackrel{\bigvee_{i=1}^{l} \Sigma \underline{p}}{\longrightarrow} \bigvee_{i=1}^{l} \Sigma S^{2 n_{i}-1}$. In particular, the map $\Sigma A \stackrel{p}{\longrightarrow} \Sigma A$ is homotopic to $\Sigma \underline{p}$, the suspension of the degree $p$ map on $A$. Now consider the composite $S^{2 n_{i}-1} \stackrel{\mu_{i}}{\longrightarrow} \Omega \Sigma A \stackrel{\Omega p}{\longrightarrow} \Omega \Sigma A$. By definition, $\mu_{i}$ is the composite $S^{2 n_{i}-1} \hookrightarrow \bigvee_{i=1}^{l} S^{2 n_{i}-1}=A \stackrel{E}{\longrightarrow} \Omega \Sigma A$. The naturality of the suspension map $E$ and $\underline{p} \simeq \Sigma \underline{p}$ therefore imply that there is a homotopy commutative diagram

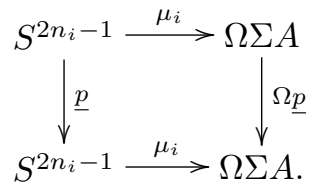

Lemma 4.1. There is a homotopy commutative diagram

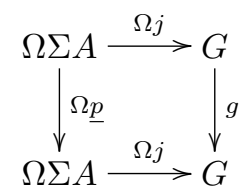

for some map $g$, which can be chosen to be an H-map.

Proof. We will show that the composite $\psi: \Omega \Sigma R \stackrel{\Omega \vartheta}{\longrightarrow} \Omega \Sigma A \stackrel{\Omega p}{\longrightarrow} \Omega \Sigma A \stackrel{\Omega j}{\longrightarrow} G$ is null homotopic. If so, then the asserted homotopy commutative diagram and the fact that $g$ can be chosen to be an $H$-map follows from Lemma 3.1. Since $\psi$ is an $H$-map, to show it is null homotopic it suffices by Theorem 2.7 to show that the composite $R \stackrel{E}{\longrightarrow} \Omega \Sigma R \stackrel{\psi}{\longrightarrow} G$ is null homotopic.

Recall that $R=\bigvee_{k=2}^{l} R_{k}$ is homotopy equivalent to a wedge of spheres, and there were more explicit breakdowns $R_{2}=R_{2,1} \vee R_{2,2}$ and $R_{3}=R_{3,1} \vee R_{3,2}$. The restriction of $\Omega \vartheta \circ E$ to $R_{k}$ for $4 \leqslant k \leqslant l$ is a wedge sum of iterated Samelson products of length $k$. By Lemma 2.4, these Samelson products compose with $\Omega j \circ \Omega p$ to give iterated Samelson products of length $k$ in $G$. These must be null homotopic since Theorem 2.11 states that the homotopy nilpotency class of $G$ is at most 3 . Thus $\psi \circ E$ is null homotopic when restricted to $\bigvee_{k=4}^{l} R_{k}$.

Next, using the fact that $\Omega p$ is an $H$-map and (10), we obtain a string of homotopies

$$
\Omega \underline{p} \circ\left\langle\mu_{i}, \mu_{j}\right\rangle \simeq\left\langle\Omega \underline{p} \circ \mu_{i}, \Omega \underline{p} \circ \mu_{j}\right\rangle \simeq\left\langle\mu_{i} \circ \underline{p}, \mu_{j} \circ \underline{p}\right\rangle \simeq\left\langle\mu_{i}, \mu_{j}\right\rangle \circ \underline{p}^{2} .
$$

By Lemma 2.10, the composite $\Omega j \circ\left\langle\mu_{i}, \mu_{j}\right\rangle$ is either null homotopic or homotopic to an element of order $p$. Thus the string of homotopies above implies that $\Omega j \circ \Omega p \circ$ $\left\langle\mu_{i}, \mu_{j}\right\rangle$ is null homotopic for all $i$ and $j$. Therefore, as the restriction of $\Omega \gamma \circ E^{-}$to $R_{2,1}$ is a wedge sum of Samelson products $\left\langle\mu_{i}, \mu_{j}\right\rangle$, the restriction of $\psi \circ E$ to $R_{2,1}$ is null homotopic. As the restriction of $\Omega \gamma \circ E$ to $R_{3,1}$ is a wedge sum of length 3 Samelson products in the $\mu_{i}$ 's, a similar argument shows that the restriction of $\psi \circ E$ to $R_{3,1}$ is null homotopic.

It remains to show that the restrictions of $\psi \circ E$ to $R_{2,2}$ and $R_{3,2}$ are null homotopic. The restriction of $\Omega \vartheta \circ E$ to $R_{2,2}$ is a wedge sum of differences $\left\langle\mu_{i}, \mu_{j}\right\rangle-b_{i, j}$ 
where $b_{i, j}$ is the composite $S^{2 n_{i}+2 n_{j}-2} \stackrel{\alpha_{1}}{\longrightarrow} S^{2 n_{i}+2 n_{j}-2 p+1} \hookrightarrow \bigvee_{i=1}^{l} S^{2 n_{i}-1}=A \stackrel{E}{\longrightarrow}$ $\Omega \Sigma A$. Since $\Omega p$ and $\Omega j$ are $H$-maps, they distribute on the left. This and the argument above for Samelson products shows that $\Omega j \circ \Omega \underline{p} \circ\left(\left\langle\mu_{i}, \mu_{j}\right\rangle-b_{i, j}\right) \simeq \Omega j \circ \Omega \underline{p} \circ b_{i, j}$. Since $\Omega \underline{p}$ is the loops on $\bigvee_{i=1}^{l} \Sigma \underline{p}$, the naturality of $E$ and the definition of $b_{i, j}$ imply that $\Omega \underline{p} \circ b_{i, j}$ is homotopic to a nonzero multiple of the composite $S^{2 n_{i}+2 n_{j}-2} \stackrel{\alpha_{1}}{\longrightarrow}$ $S^{2 n_{i}+2 n_{j}-2 p+1} \stackrel{\underline{p}}{\longrightarrow} S^{2 n_{i}+2 n_{j}-2 p+1} \hookrightarrow \bigvee_{i=1}^{l} S^{2 n_{i}-1}=A \stackrel{E}{\longrightarrow} \Omega \Sigma A$. There are two cases. First, if $2 n_{i}+2 n_{j}-2 p+1 \geqslant 5$ then $\alpha_{1}$ is a suspension, so $p \circ \alpha_{1} \simeq \alpha_{1} \circ p$. Since $\alpha_{1}$ has order $p$, we obtain that $\alpha_{1} \circ \underline{p}$ is null homotopic. Second, if $2 n_{i}+2 n_{j}-2 p+1=3$ then $\alpha_{1}$ is not a suspension. But the double suspension $S^{3} \stackrel{E^{2}}{\longrightarrow}$ $\Omega^{2} S^{5}$ induces an isomorphism in homotopy groups in dimensions $\leqslant 4 p-4$. Therefore the composite $S^{2 p} \stackrel{\underline{p}}{\longrightarrow} S^{2 p} \stackrel{\alpha_{1}}{\longrightarrow} S^{3}$ is null homotopic since its double suspension is. Hence, in either case, $\Omega \underline{p} \circ b_{i, j}$ and therefore $\Omega j \circ \Omega \underline{p} \circ\left(\left\langle\mu_{i}, \mu_{j}\right\rangle-b_{i, j}\right)$ are null homotopic. Consequently, the restriction of $\psi \circ E$ to $R_{2,2}$ is null homotopic.

The argument to show that the restriction of $\psi \circ E$ to $R_{3,2}$ is null homotopic is similar. In this case, the difference maps are $\left\langle\mu_{t},\left\langle\mu_{i}, \mu_{j}\right\rangle\right\rangle-c_{t, i, j}$, where, if $c_{t, i, j}$ is nontrivial, then it is homotopic to the composite $S^{4 p-3} \stackrel{\alpha_{1}}{\longrightarrow} S^{2 p} \stackrel{\alpha_{1}}{\longrightarrow} S^{3}$, and this composite composes trivially with the degree $p$ map on $S^{3}$ since we have just seen that $S^{2 p} \stackrel{\alpha_{1}}{\longrightarrow} S^{3}$ does.

What Lemma 4.1 does not do is show that the map $g$ is homotopic to the $p^{\text {th }}$ power map on $G$. To show this we bring in the Hilton-Milnor Theorem. For a space $X$ and an integer $m$, let $X^{(m)}$ be the $m$-fold smash of $X$ with itself. Let $X_{i}, 1 \leqslant i \leqslant p$, be path-connected spaces and consider the wedge $\Sigma X_{1} \vee \cdots \vee \Sigma X_{p}$. Let $i_{j}: \Sigma X_{j} \longrightarrow$ $\Sigma X_{1} \vee \cdots \vee \Sigma X_{p}$ be the inclusion of the $j^{t h}$-summand into the wedge. The HiltonMilnor Theorem states that there is a homotopy equivalence

$$
\Psi: \prod_{i=1}^{p} \Omega \Sigma X_{i} \times \prod_{\alpha \in \mathcal{I}} \Omega\left(\Sigma X_{1}^{(\alpha(1))} \wedge \cdots \wedge X_{p}^{(\alpha(p))}\right) \longrightarrow \Omega\left(\Sigma X_{1} \vee \cdots \vee \Sigma X_{p}\right)
$$

where: (i) the index set $\mathcal{I}$ runs over an additive basis of the free Lie algebra $L\left\langle u_{1}, \ldots, u_{p}\right\rangle$, but excludes the generators $u_{i}$; (ii) $\alpha \in \mathcal{I}$ corresponds to a bracket in $L\left\langle u_{1}, \ldots, u_{p}\right\rangle$ and each $\alpha(i)$ counts the number of appearances of $u_{i}$ in that bracket; (iii) the space $\Sigma X_{1}^{(\alpha(1))} \wedge \cdots \wedge X^{(\alpha(p))}$ is mapped to $\Sigma X_{1} \vee \cdots \vee \Sigma X_{(p)}$ by taking the Whitehead product $\bar{w}_{\alpha}$ of the maps $i_{1}, \ldots, i_{p}$ corresponding to the bracket $\alpha$; and (iv) the map $\Psi$ is formed by taking the product of $\Omega i_{j}$ for $1 \leqslant i \leqslant p$ and $\Omega \bar{w}_{\alpha}$ for each $\alpha \in \mathcal{I}$. The composite of $\Psi^{-1}$ with the projection onto a factor defines a map

$$
\bar{H}_{\alpha}: \Omega\left(\Sigma X_{1} \vee \cdots \vee \Sigma X_{p}\right) \longrightarrow \Omega\left(\Sigma X_{1}^{\left(\alpha_{1}\right)} \wedge \cdots \wedge X_{p}^{\left(\alpha_{p}\right)}\right) .
$$

Now suppose that $X_{i}=X$ for each $1 \leqslant i \leqslant p$. Let $\delta: \Sigma X \longrightarrow \Sigma X \vee \Sigma X$ be the comultiplication determined by the suspension. Iterating, we obtain a map $\sigma: \Sigma X \longrightarrow$ $\bigvee_{i=1}^{p} \Sigma X$. Note that the order of the iteration is irrelevant as the suspension co- $H$ structure is homotopy coassociative. For $\alpha \in \mathcal{I}$, let $H_{\alpha}$ be the Hilton-Hopf invariant, defined by the composite

$$
H_{\alpha}: \Omega \Sigma X \stackrel{\Omega \sigma}{\longrightarrow} \Omega\left(\bigvee_{i=1}^{p} \Sigma X\right) \stackrel{\bar{H}_{\alpha}}{\longrightarrow} \Omega\left(\Sigma X^{(\alpha(1)+\cdots+\alpha(p))}\right)
$$


and let $w_{\alpha}$ be the composite

$$
w_{\alpha}: \Sigma X^{(\alpha(1)+\cdots+\alpha(p))} \stackrel{\bar{w}_{\alpha}}{\longrightarrow} \bigvee_{i=1}^{p} \Sigma X \stackrel{\nabla}{\longrightarrow} \Sigma X
$$

where $\nabla$ is the fold map. The degree $p$ map on $\Sigma X$ is the composite $\Sigma X \stackrel{\sigma}{\longrightarrow}$ $\bigvee_{i=1}^{p} \Sigma X \stackrel{\nabla}{\longrightarrow} \Sigma X$. Thus $\Omega \underline{p}$ is the composite

$$
\Omega \Sigma X \stackrel{\Omega \sigma}{\longrightarrow} \Omega\left(\bigvee_{i=1}^{p} \Sigma X\right) \stackrel{\Omega \nabla}{\longrightarrow} \Omega \Sigma X .
$$

Applying the iterated Hilton-Milnor Theorem to $\Omega\left(\bigvee_{i=1}^{p} \Sigma X\right)$, we obtain the formula

$$
\Omega \underline{p} \simeq p+\sum_{\alpha \in \mathcal{I}} \Omega w_{\alpha} \circ H_{\alpha} .
$$

Equation (11) has been well studied, particularly by Barratt [Ba], and it may be due to him.

Let $\mathcal{I}_{t} \subset \mathcal{I}$ be the index set corresponding to the length $t$ Whitehead products. Barratt $[\mathbf{B a}, 6.9]$ proved the following. Note that Barratt's statement is phrased in terms of homotopy groups rather than spaces, but the proof translates to spaces without any change.

Lemma 4.2. If $X$ is a suspension and $t<p$, there is a subset $\mathcal{I}_{t}^{\prime} \subset \mathcal{I}_{t}$ such that

$$
\sum_{\alpha \in \mathcal{I}_{t}} \Omega w_{\alpha} \circ H_{\alpha} \simeq \sum_{\alpha^{\prime} \in \mathcal{I}_{t}^{\prime}} a_{t} \Omega w_{\alpha^{\prime}} \circ H_{\alpha^{\prime}}
$$

where $a_{t}$ is an integer divisible by $p$.

We will apply Lemma 4.2 to see what happens when $\Omega \underline{p}-p$ on $\Omega \Sigma A$ is composed into $G$. First a preliminary lemma is needed to gauge the effect of composing $\Omega w_{\alpha}$ on $\Omega \Sigma A$ into $G$.

Lemma 4.3. If the Whitehead product $w_{\alpha}$ is of length $\geqslant 4$, then the composite

$$
\Omega \Sigma A^{(\alpha(1)+\cdots+\alpha(p))} \stackrel{\Omega w_{\alpha}}{\longrightarrow} \Omega \Sigma A \stackrel{\Omega j}{\longrightarrow} G
$$

is null homotopic.

Proof. By Theorem 2.7, the homotopy class of the $H$-map $\Omega j \circ \Omega w_{\alpha}$ is determined by the restriction $\Omega j \circ \Omega w_{\alpha} \circ E$. Since $w_{\alpha}$ is a Whitehead product of length $t \geqslant 4$, the restriction $\Omega w_{\alpha} \circ E$ is a Samelson product of length $t$. Lemma 2.6 then implies that $\Omega j \circ \Omega w_{\alpha} \circ E$ is a Samelson product of length $t$. By Theorem 2.11, the homotopy nilpotency class of $G$ is at most 3 , so $\Omega j \circ \Omega w_{\alpha} \circ E$ is null homotopic. Hence $\Omega j \circ \Omega w_{\alpha}$ is null homotopic.

Proposition 4.4. If $p \geqslant 5$ then the composite $\Omega \Sigma A \stackrel{\Omega \underline{p}-p}{\longrightarrow} \Omega \Sigma A \stackrel{\Omega j}{\longrightarrow} G$ is null homotopic. 
Proof. Since $\Omega j$ is an $H$-map, it distributes on the left. Thus

$$
\Omega j \circ(\Omega \underline{p}-p) \simeq \Omega j \circ\left(\sum_{\alpha \in \mathcal{I}} \Omega w_{\alpha} \circ H_{\alpha}\right) \simeq \sum_{\alpha \in \mathcal{I}} \Omega j \circ \Omega w_{\alpha} \circ H_{\alpha} .
$$

Lemma 4.3 implies that $\Omega j \circ \Omega w_{\alpha}$ is null homotopic if $w_{\alpha}$ is an iterated Whitehead product of length $t \geqslant 4$. So we are reduced to showing that $\sum_{\alpha \in \mathcal{I}_{t}} \Omega j \circ \Omega w_{\alpha} \circ H_{\alpha}$ is null homotopic for $t \in\{2,3\}$.

Since $A$ is a wedge of 2 -connected spheres, it is a suspension. If $p \geqslant 5$, then Lemma 4.2 implies that for $t \in\{2,3\}$,

$$
\sum_{\alpha \in \mathcal{I}_{t}} \Omega j \circ \Omega w_{\alpha} \circ H_{\alpha} \simeq \sum_{\alpha^{\prime} \in \mathcal{I}_{t}^{\prime}} p \cdot a_{t}^{\prime} \Omega j \circ \Omega w_{\alpha^{\prime}} \circ H_{\alpha^{\prime}}
$$

for some integer $a_{t}^{\prime}$. (Note that if $p=3$, Theorem 2.11 implies that $\operatorname{nil}(G)=3$ for $G$ equal to $S U(2)$ (equivalently, $S p(1)$ or $\operatorname{Spin}(3)$ ) and $S U(3)$, implying that the appeal to Lemma 4.2 is no longer valid.)

Consider the composite $\Omega \Sigma A^{(2)} \stackrel{\Omega w_{q^{\prime}}}{\longrightarrow} \Omega \Sigma A \stackrel{\Omega j}{\longrightarrow} G$, where $w_{\alpha^{\prime}}$ is a Whitehead product of length 2. The restriction $\Omega w_{\alpha^{\prime}} \circ E$ is a Samelson product of length 2. Since $A$ is a wedge of spheres, so is $A^{(2)}$, and under this equivalence $\Omega w_{\alpha^{\prime}} \circ E$ is homotopic to a wedge sum of length 2 Samelson products of the form $\left\langle\mu_{i}, \mu_{j}\right\rangle$. Thus $\Omega j \circ \Omega w_{\alpha^{\prime}} \circ E$ is homotopic to a wedge sum of Samelson products of the form $\left\langle x_{i}, x_{j}\right\rangle$. By Lemma 2.10, $\left\langle x_{i}, x_{j}\right\rangle \circ \underline{p}$ is null homotopic. Thus $\Omega j \circ \Omega w_{\alpha^{\prime}} \circ E \circ \underline{p}$, is null homotopic, where $\underline{p}$ is the degree $p$ map on $A^{(2)}$. By Lemma 2.7, this implies that the composite of loop maps $\Omega \Sigma A^{(2)} \stackrel{\Omega \Sigma \underline{p}}{\longrightarrow} \Omega \Sigma A^{(2)} \stackrel{\Omega w_{\alpha^{\prime}}}{\longrightarrow} \Omega \Sigma A \stackrel{\Omega j}{\longrightarrow} G$ is null homotopic. This is not quite what we want: we are aiming at a null homotopy for $\Omega j \circ \Omega w_{\alpha^{\prime}} \circ p$. However, we can apply (11) to the difference $\Omega \Sigma \underline{p}-p$ on $\Omega \Sigma A^{(2)}$. We obtain

$$
\Omega \Sigma \underline{p}-p \simeq \sum_{\beta \in \mathcal{J}} \Omega w_{\beta} \circ H_{\beta}
$$

Observe that if $w_{\beta}$ is Whitehead product on $\Sigma A^{(2)}$ of length $t$, then the composite $\Sigma A^{(2 t)} \stackrel{w_{\beta}}{\longrightarrow} \Sigma A^{(2)} \stackrel{w_{\alpha^{\prime}}}{\longrightarrow} \Sigma A$ is a Whitehead product of length $2 t$. Thus, as $t \geqslant 2$ and the homotopy nilpotency class of $G$ is at most 3, Lemma 4.3 implies that the composite $\Omega \Sigma A^{(2 t)} \stackrel{\Omega w_{\beta}}{\longrightarrow} \Omega \Sigma A^{(2)} \stackrel{\Omega w_{\alpha^{\prime}}}{\longrightarrow} \Omega \Sigma A \stackrel{\Omega j}{\longrightarrow} G$ is null homotopic for every $\beta \in \mathcal{J}$. Hence there is a string of homotopies

$$
\begin{aligned}
\Omega j \circ \Omega w_{\alpha^{\prime}} \circ(\Omega \Sigma \underline{p}-p) & \simeq \Omega j \circ \Omega w_{\alpha^{\prime}} \circ\left(\sum_{\beta \in \mathcal{J}} \Omega w_{\beta} \circ H_{\beta}\right) \\
& \simeq\left(\sum_{\beta \in \mathcal{J}} \Omega j \circ \Omega w_{\alpha^{\prime}} \circ \Omega w_{\beta} \circ H_{\beta}\right) \\
& \simeq * .
\end{aligned}
$$

Since we have also shown that $\Omega i \circ \Omega w_{\alpha^{\prime}} \circ \Omega \Sigma p$ is null homotopic, we therefore obtain a null homotopy for $\Omega i \circ \Omega w_{\alpha^{\prime}} \circ p$. This is true for every Whitehead product $w_{\alpha^{\prime}}$ of length 2 , so $\sum_{\alpha^{\prime} \in \mathcal{I}_{2}^{\prime}} p \cdot a_{t}^{\prime} \Omega i \circ \Omega w_{\alpha^{\prime}} \circ H_{\alpha}$, is null homotopic.

A similar argument shows that $\sum_{\alpha^{\prime} \in \mathcal{I}_{3}^{\prime}} p \cdot a_{t}^{\prime} \Omega i \circ \Omega w_{\alpha^{\prime}} \circ H_{\alpha,}$ is null homotopic when $p \geqslant 5$.

Since $\Omega j$ is an $H$-map, it distributes on the left, implying that $\Omega j \circ(p-\Omega \underline{p}) \simeq$ $\Omega j \circ p-\Omega j \circ \Omega \underline{p}$. Thus Proposition 4.4 immediately implies the following. 
Corollary 4.5. If $p \geqslant 5$, then the composite $\Omega \Sigma A \stackrel{p}{\longrightarrow} \Omega \Sigma A \stackrel{\Omega j}{\longrightarrow} G$ is homotopic to the composite $\Omega \Sigma A \stackrel{\Omega p}{\longrightarrow} \Omega \Sigma A \stackrel{\Omega j}{\longrightarrow} G$.

Proof of Theorem 1.2. By Lemma 2.1, the map $\Omega \Sigma A \stackrel{\Omega j}{\longrightarrow} G$ has a right homotopy inverse $s: G \longrightarrow \Omega \Sigma A$. Combining Lemma 4.1 and $s$, we obtain a homotopy commutative diagram

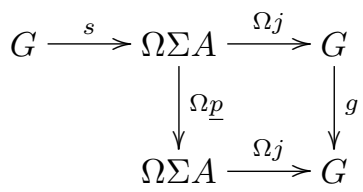

where $g$ is an $H$-map. Since $s$ is a right homotopy inverse of $\Omega j$, the upper direction around the diagram is homotopic to $g$. As $p \geqslant 5$, by Corollary 4.5 the composite around the lower direction around the diagram is homotopic to $\Omega j \circ p \circ s$. Since $p$ commutes with $H$-maps, this in turn is homotopic to $p \circ \Omega j \circ s \simeq p$. Hence $g \simeq p$, and so the $p^{t h}$-power map on $G$ is an $H$-map.

\section{References}

[AC] M. Arkowitz and C.R. Curjel, On the maps of $H$-spaces, Topology 6 (1967), 137-148.

[Ba] M.G. Barratt, Spaces of finite characteristic, Q. J. Math. Oxf. II Ser. 11 (1960), 124-136.

[Bo] R. Bott, A note on the Samelson product in the classical groups, Comment. Math. Helv. 34 (1960), 245-256.

[CMN] F.R. Cohen, J.C. Moore, and J.A. Neisendorfer, Torsion in homotopy groups, Ann. Math. (2) 109 (1979), 121-168.

[CN] F.R. Cohen and J.A. Neisendorfer, A construction of $p$-local $H$-spaces, Algebraic topology, Aarhus 1982 (Aarhus, 1982), Lecture Notes in Math., vol. 1051, Springer, Berlin, 1984, pp. 351-359.

[F] E.M. Friedlander, Exceptional isogenies and the classifying spaces of simple Lie groups, Ann. Math. (2) 101 (1975), 510-520.

[Gr] B. Gray, Homotopy commutativity and the EHP sequence. Algebraic topology (Evanston, IL, 1988), Contemp. Math., vol. 96, Amer. Math. Soc., Providence, RI, 1989, 181-188.

[G1] J. Grbić, Universal homotopy associative, homotopy commutative $H$-spaces and the EHP spectral sequence, Math. Proc. Cambridge Philos. Soc., 140 (2006), no. 3, 377-400.

[G2] J. Grbić, Universal spaces of two-cell complexes and their exponent bounds, Q. J. Math. 57 (2006), 355-366.

[GT] J. Grbić and S. Theriault, Self-maps of low rank Lie groups at odd primes, Can. Math. J. 62 (2010), 284-304.

[HK] H. Hamanaka and A. Kono, A note on Samelson products and mod- $p$ cohomology of classifying spaces of the exceptional Lie groups, Topology Appl. 157 (2010), 393-400. 
[J] I.M. James, Reduced product spaces, Ann. Math. (2) 62 (1955), 170-197.

[KK] S. Kaji and D. Kishimoto, Homotopy nilpotency in $p$-regular loop spaces, Math. Z. 264 (2010), 209-224.

[KKT] D. Kishimoto, A. Kono and S. Theriault, Homotopy commutativity in $p$ localized gauge groups, Proc. Roy. Soc. Edin. 143 (2013), 851-870.

[M1] C.A. McGibbon, Multiplicative properties of power maps I, Q. J. Math. Oxf. II Ser. 31 (1980), 341-350.

[M2] C.A. McGibbon, Homotopy commutativity in localized groups, Amer. J. Math. 106 (1984), 665-687.

[T1] S.D. Theriault, The $H$-structure of low rank torsion free $H$-spaces, Q. J. Math. Oxf. II Ser. 56 (2005), 403-415.

[T2] S.D. Theriault, The odd primary $H$-structure of low rank Lie groups and its application to exponents, Trans. Am. Math. Soc. 359 (2007), 4511-4535.

[T3] S.D. Theriault, Odd primary homotopy decompositions of gauge groups, Algebr. Geom. Top. 10 (2010), 535-564.

Stephen Theriault S.D.Theriault@soton.ac.uk

School of Mathematics, University of Southampton, Southampton SO17 1BJ, United Kingdom 